\title{
miR-135b levels in the peripheral blood serve as a marker associated with acute ischemic stroke
}

\author{
SHA YANG ${ }^{1}$, XINYU ZHAN ${ }^{1}$, MIN HE $^{1}$, JINGJING WANG $^{2}$ and XUEMEI QIU ${ }^{3}$ \\ ${ }^{1}$ Department of Clinical Laboratory Medicine, Southwest Hospital, Third Military Medical University, \\ Chongqing 400038; ${ }^{2}$ Vasculocardiology Department, The 903rd Hospital of PLA, Hangzhou, Zhejiang 310013; \\ ${ }^{3}$ Department of Clinical Laboratory Medicine, The 80th Army Hospital of PLA, Weifang, Shandong 261021, P.R. China
}

Received August 12, 2019; Accepted February 26, 2020

DOI: $10.3892 / \mathrm{etm} .2020 .8628$

\begin{abstract}
The protective role of microRNA (miR)-135b in cerebral neurons has been previously identified. However, to the best of our knowledge, the involvement of miR-135b in acute ischemic stroke has yet to be elucidated. The present study aimed to investigate the expression profile of miR-135b in peripheral blood obtained from patients with acute ischemic stroke. A total of 76 patients with acute ischemic stroke were selected as the case group, which included 33 cases of aorta atheromatous plague, 19 cases of cardioembolism, 16 cases of small arterial occlusion and 8 cases with unknown causes. In addition, 60 healthy subjects were selected as the control group. Reverse transcription-quantitative PCR was used to measure the expression of miR-135b in the peripheral blood of the patients. The National Institutes of Health Stroke Scale (NIHSS) score was used to evaluate the severity of acute ischemic stroke. The relationship between miR-135b levels and acute stroke was subsequently analyzed. The expression of miR-135b in the peripheral blood of the case group was found to be significantly higher compared with that in the control group. By contrast, the expression levels of miR-135b in the case group did not differ significantly between the different etiology types of acute ischemic stroke. In addition, a significant positive correlation was observed between levels of miR-135b expression and NIHSS scores. Further analysis demonstrated that hypertension, hyperglycemia, platelet count, international normalized ratio and miR-135b were risk factors for acute ischemic stroke. Based on bioinformatics analysis, a conserved binding site for miR-135b was identified in the 3 -untranslated region of the transient receptor potential cation channel subfamily C member 6 (TRPC6). Dual luciferase
\end{abstract}

Correspondence to: Dr Sha Yang, Department of Clinical Laboratory Medicine, Southwest Hospital, Third Military Medical University, 30 Gaotanyan Street, Chongqing 400038, P.R. China E-mail: shayang0714@163.com

Key words: acute ischemic stroke, microRNA-135b, National Institutes of Health Stroke Scale, transient receptor potential cation channel subfamily $\mathrm{C}$ member 6 reporter and western blot analysis showed that TRPC6 was a target gene of miR-135b. In conclusion, data from the present study suggest that elevated expression of miR-135b in the peripheral blood of patients with acute ischemic stroke is closely associated with disease severity.

\section{Introduction}

Stroke, which is also known as cerebral infarction, is the sudden deprivation of blood supply in the brain (1). In addition to having $6.1 \%$ mortality, stroke is also associated with a high risk of disability and recurrence around the world, accounting for 3\% of the total health expenditure (2-4). This condition is mainly divided into two categories, ischemic stroke and hemorrhagic stroke, of which the former is the most prevalent, accounting for $\sim 80 \%$ of total cases (5). Common risk factors for this disease include high blood pressure, smoking, hyperlipidemia, obesity, diabetes, coronary heart disease and a family history of stroke (6). Among these factors, carotid stenosis and occlusion as a result of atherosclerosis are main risk factors for stroke (7).

In recent years, studies have demonstrated that microRNAs (miRs/miRNAs) serve important roles in a number of diseases, including vascular disease, metabolic disease and cancer $(8,9)$. Recent studies showed that miRNAs can potentially serve as novel biomarkers and therapeutic targets for a variety of pathological conditions (5). miRNAs have a wide range of biological functions and remain stable in bodily fluids including blood, urine and amniotic fluid $(10,11)$. Previous reports found significant differences in the expression of several miRNAs, including miR-30a, miR-126 and Let-7b, in the peripheral blood of patients with stroke at different time points $(12,13)$. It was previously reported that serum miR-21 and miR-221 levels in addition to the peripheral blood levels of miR-210, miR-200c-3p, miR-99b-5p and miR-150-5p could be used as biomarkers for stroke $(14,15)$.

The neuroprotective role of miR-135b in SH-SY5Y cells and stress-enhanced memory has been previously demonstrated $(16,17)$. In Parkinson's disease (PD), the protective role of miR- $135 b$ is achieved by targeting GSK-3 $\beta$ in SH-SY5Y cells intoxicated with 1-methyl-4-phenyl-pyridinium (16). Additionally, overexpression of miR-135b-5p in the basolateral amygdala complex of stress-resilient animals increased remote 
fear memory expression and contributed to spontaneous renewal 14 days after death (17). These observations suggested the potentially important role of miR-135b in cerebral neurons. However, the effect of miR-135b on acute ischemic stroke (AIS) remains to be fully elucidated.

Therefore, the present study aimed to measure the expression of miR-135b in the peripheral blood of patients with AIS and to analyze the relationship between miR-135b expression and disease etiology and severity to assess the occurrence of miR-135b in AIS.

\section{Materials and methods}

General information. A total of 76 patients with AIS who were treated at Southwest Hospital (Chongqing, China) between January 2018 and April 2018 were selected as the case group (IS group) based on AIS guidelines (18). The inclusion criteria were as follows: i) Brain CT/MRI examination revealing ischemic lesions; ii) numbness or weakness present in one limb or face and speech difficulty; and iii) sudden onset of stroke, which necessitated emergency admission to the Southwest Hospital. The exclusion criteria were as follows: i) Hemorrhagic stroke, brain trauma, hypertensive encephalopathy and encephalitis; ii) a previous history of stroke and head trauma surgery; iii) other diseases in the nervous and vascular system, such as meningitis, myelitis, epilepsy and dementia; and iv) malignant brain tumors or multiple sclerosis. According to the The Trial of Org 10172 in Acute Stroke Treatment (TOAST) classification etiology, the 76 patients in the IS group were divided further into 33 cases of aorta atheromatous plague, 19 cases of cardioembolism, 16 cases of small arterial occlusion and 8 cases with unknown causes. In addition, 60 healthy subjects in Southwest Hospital were selected as the control group within the same time period. Informed and signed consent was obtained from both groups of subjects and their families. The present study was approved by the Ethics Committee of Southwest Hospital (approval no. SH-20160986).

In the IS group, $5 \mathrm{ml}$ peripheral blood was collected on the day of admission and 14 days after admission, whilst $5 \mathrm{ml}$ peripheral blood was obtained from the control group (NC group) on the day of physical examination. Subsequently, the serum samples were kept at $-80^{\circ} \mathrm{C}$ until further use. Sex, age, blood glucose, blood pressure and total biochemical indicators, including total cholesterol, glycerol, platelet count and blood zymogen time/international normalized ratio (INR), were routinely recorded at 8:00 a.m. every day for 14 days. The severity of disease affliction was evaluated according to the National Institutes of Health Stroke Scale (NIHSS) (19) by a professional physician. The score ranged from 0 to 42 , with higher scores positively associated with the severity of neurological damage.

Serum total RNA extraction. Extraction of total RNA from serum was performed via enrichment using an RNeasy Micro kit (cat. no. 74004; Qiagen GmbH) according to the kit instructions. RNA concentration and purity were determined using a BioPhotometer ${ }^{\mathrm{TM}}$ Plus nucleic acid protein analyzer (Eppendorf) where samples with a total RNA absorbance of 1.8-2.0 were used for subsequent experiments. A total of $10 \mu \mathrm{l}$
RNA was used to assess RNA quality by denaturing agarose gel electrophoresis. RNA samples were stored at $-80^{\circ} \mathrm{C}$.

Reverse transcription (RT)-quantitative PCR (RT-qPCR). The RT reaction for mature miR-135b was performed using a PrimeScript RT reagent kit (Takara Bio, Inc.) based on the manufacturer's protocols. RT products were stored at $-20^{\circ} \mathrm{C}$. The subsequent qPCR mix was made up to $20 \mu \mathrm{l}$ with the following: fluorescent quantitative probe reaction mixture (10 $\mu \mathrm{l})$ (2X ProbeMixture), $1 \mu \mathrm{l}$ template cDNA, $1 \mu \mathrm{l} 20 \mathrm{X}$ miRNA probe, $1 \mu \mathrm{l}$ amplification primer premix and $7 \mu \mathrm{l}$ RNase-free $\mathrm{ddH}_{2} \mathrm{O}$. The following thermocycling conditions were used for the qPCR: Initial denaturation at $95^{\circ} \mathrm{C}$ for $10 \mathrm{~min}$, followed by 45 cycles of $95^{\circ} \mathrm{C}$ for $15 \mathrm{sec}$ and $60^{\circ} \mathrm{C}$ for $1 \mathrm{~min}$. U6 was used as external reference for the standardization of extraction and reverse transcription. Relative mRNA expression was normalized to U6 using the $2^{-\Delta \Delta C q}$ method (20). The following primer pairs were used for the PCR: U6, forward, 5'-GCTTCGGCAGCACATATACTAAAAT-3' and reverse, 5'-CGCTTCACGAATTTGCGTGTCAT-3'; miR-135b-5p forward, 5'-GGTATGGCTTTTCATTCCT-3' and reverse, 5'-CAGTGCGTGTCGTGGAGT-3'.

Cell culture. PC12 cells were purchased from American Type Culture Collection and cultured as described previously (21).

Transient transfection. miR-135b mimic (5'-UAUGGC UUUUCAUUCCUAUGUGA-3'), mimic control (5'-UAU AUCG UGUUAUUAGCGUUCCU-3') (both from Shanghai GenePharma Co., Ltd.), inhibitor (5'-UCACAUAGG AAUGAAAAGCCAUA-3') or inhibitor control (5'-UUCAUC GUGUUAUUAGCGUUCCU-3') was transfected into PC12 cells using Lipofectamine ${ }^{\circledR} 2000$ (Invitrogen; Thermo Fisher Scientific, Inc.) according to the manufacturer's protocols. In brief, PC12 cells were cultured at a density of $1 \times 10^{6}$ cells/well and miR-135 mimic, mimic control, inhibitor or inhibitor control were transfected after $24 \mathrm{~h}$ at a final concentration of $20 \mathrm{nM}$. At $48 \mathrm{~h}$ after cell transfection, transfection efficiency was detected using RT-qPCR.

Dual luciferase assay. TargetScan (http://www.targetscan. org/vert_72/) analysis identified a conserved binding site for miR-135b in the 3'-untranslated region (3'-UTR) of transient receptor potential cation channel subfamily $\mathrm{C}$ member 6 (TRPC6). The target open reading frame from the genomic DNA of PC12 cells was then cloned into a pmirGLO plasmid (Promega Corporation). PC12 cells were cultured at a density of $1 \times 10^{6}$ cells/well in a six-well plate. Subsequently, the miR-135b mimic + pmirGLO-TRPC6-3'UTR or NC + pmirGLO was transfected into PC12 cells at a final concentration of $20 \mathrm{nM}$ for $48 \mathrm{~h}$ using Lipofectamine ${ }^{\circledR} 2000$ (Invitrogen; Thermo Fisher Scientific, Inc.) according to the manufacturer's protocols. The cells were then collected, where the relative luciferase units were determined using a Dual-Luciferase ${ }^{\circledR}$ reporter assay kit (Promega Corporation) according to the manufacturer's protocols. Firefly luciferase activity was normalized to Renilla luciferase activity.

Western blotanalysis.PC12 cells were collected and treated with RIPA buffer (Beijing Solarbio Science \& Technology Co., Ltd.) 
Table I. General clinical data for patients with AIS and healthy controls.

\begin{tabular}{|c|c|c|c|}
\hline Indicator & Healthy controls $(n=60)$ & AIS patients $(n=76)$ & P-value \\
\hline Sex (male/female) & $41 / 19$ & $55 / 21$ & 0.621 \\
\hline Average age (years) & $53.72 \pm 11.26$ & $54.68 \pm 10.98$ & 0.815 \\
\hline Smoking $[\mathrm{n}(\%)]$ & $27(45.00)$ & $39(51.35)$ & 0.464 \\
\hline Drinking $[\mathrm{n}(\%)]$ & $21(35.00)$ & $33(43.42)$ & 0.319 \\
\hline Systolic blood pressure (mmHg) & $121.15 \pm 10.28$ & $152.62 \pm 10.75$ & $<0.001$ \\
\hline Diastolic blood pressure (mmHg) & $79.84 \pm 8.63$ & $98.52 \pm 6.85$ & $<0.001$ \\
\hline Previous cerebrovascular event $[\mathrm{n}(\%)]$ & 0 & $25(32.9)$ & $<0.001$ \\
\hline Family cerebrovascular event [n (\%)] & $0(0.0)$ & $18(23.7)$ & $<0.001$ \\
\hline Homocysteine level ( $\mu$ mol/l) & $11.46 \pm 1.85$ & $22.56 \pm 6.35$ & 0.043 \\
\hline Uric acid $(\mu \mathrm{mol} / \mathrm{l})$ & $235.56 \pm 73.11$ & $359.03 \pm 96.71$ & 0.031 \\
\hline $\mathrm{FBG}(\mathrm{mol} / \mathrm{l})$ & $5.38 \pm 1.32$ & $7.25 \pm 1.96$ & $<0.001$ \\
\hline $\mathrm{TC}(\mathrm{mmol} / \mathrm{l})$ & $5.12 \pm 1.38$ & $5.24 \pm 1.76$ & 0.589 \\
\hline $\mathrm{TG}(\mathrm{mmol} / \mathrm{l})$ & $1.46 \pm 0.35$ & $1.44 \pm 0.48$ & 0.762 \\
\hline Platelet count $\left(\mathrm{x} 10^{9} / 1^{3}\right)$ & $215.82 \pm 33.56$ & $175.78 \pm 26.84$ & $<0.001$ \\
\hline INR & $1.12 \pm 0.26$ & $2.63 \pm 0.72$ & $<0.001$ \\
\hline NIHSS score & $\mathrm{N} / \mathrm{A}$ & $18.82 \pm 5.14$ & N/A \\
\hline Intravenous thrombolytic therapy [n (\%)] & $0(0.0)$ & $26(34.2)$ & N/A \\
\hline
\end{tabular}

Data were represented as the mean \pm SD unless otherwise stated. AIS, acute ischemic stroke; FBG, fasting blood glucose; TC, total cholesterol; TG, triglyceride; INR, international normalized ratio; NIHSS, National Institutes of Health Stroke Scale.

containing $1 \%(\mathrm{v} / \mathrm{v})$ phenylmethylsulfonyl fluoride (Beijing Solarbio Science \& Technology Co., Ltd.), $0.3 \%$ (v/v) protease inhibitor (Sigma-Aldrich; Merck KGaA) and $0.1 \%$ (v/v) phosphorylated proteinase inhibitor (Sigma-Aldrich; Merck KGaA). Western blots were performed as previously described (22). Membranes were incubated with primary antibodies against TRPC6 (cat. no. ab62461; 1:1,000; Abcam) and anti-GAPDH (cat. no. 2118; 1:5,000; Cell Signaling Technology, Inc.) overnight at $4^{\circ} \mathrm{C}$. Membranes were subsequentlyincubated with horseradish peroxidase-conjugated goat anti-rabbit immunoglobulin G (cat. no. ZB-2301; 1:5,000; Beijing Zhongshan Jinqiao Biotechnology Co., Ltd.) for $2 \mathrm{~h}$ at room temperature, followed by three washes with TBS-Tween-20. Enhanced chemiluminescence (ECL; EMD Millipore) was used to determine protein concentrations according to the manufacturer's protocol. Protein signal was detected using a Super ECL Plus kit (Nanjing KeyGen Biotech Co., Ltd.). Relative protein expression was normalized to GAPDH. All experiments were repeated three times. ImageJ v1.43b software (National Institutes of Health) was used for densitometric analysis.

Statistical analysis. Data were represented as the mean \pm SD. Statistical analysis was performed on SPSS 20.0 (IBM Corp.). Two-tailed unpaired Student's t-test was used for comparisons between two groups. One-way ANOVA multiple comparison test followed by Tukey's post hoc test was used for comparisons between $>2$ groups. Chi square test was performed for comparing categorical variables of the clinical data. Receiver operating characteristic (ROC) curves was used to assess miR-135b as a diagnostic biomarker, where the area under the curve (AUC) was calculated. Correlation between miR-135b expression and NIHSS scores was analyzed using the Pearson correlation coefficient (r) method; logistic regression analysis was used to explore the risk factors affecting AIS. Kaplan-Meier analysis followed by log-rank test was used to analyze the overall survival rate based the levels of miR-135b in peripheral blood. $\mathrm{P}<0.05$ was considered to indicate a statistically significant difference.

\section{Results}

Clinical data. No significant differences in sex, mean age, smoking, alcohol consumption, total cholesterol and triglyceride levels were found between the IS and control groups (Table I). Systolic blood pressure, diastolic blood pressure and fasting blood glucose in the IS group were found to be significantly higher compared with those in the control group (Table I). The platelet count in the IS group was significantly lower compared with that in the control group, whilst the prothrombin time/international normalized ratio (INR) was significantly higher in the IS group compared with that in the control group (Table I). The NIHSS score was found to be $18.82 \pm 5.14$ for the IS group (Table I).

Expression levels of peripheral blood miR-135b in patients with IS and healthy controls. RT-qPCR was used to measure the expression levels of miR-135b in the peripheral blood of IS and control groups. Serum miR-135b expression levels was revealed to be significantly higher in the IS group (4.20 \pm 2.35$)$

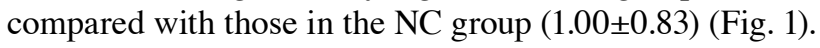

Peripheral blood miR-135b expression levels among groups with different etiologies of AIS within the IS group. No 


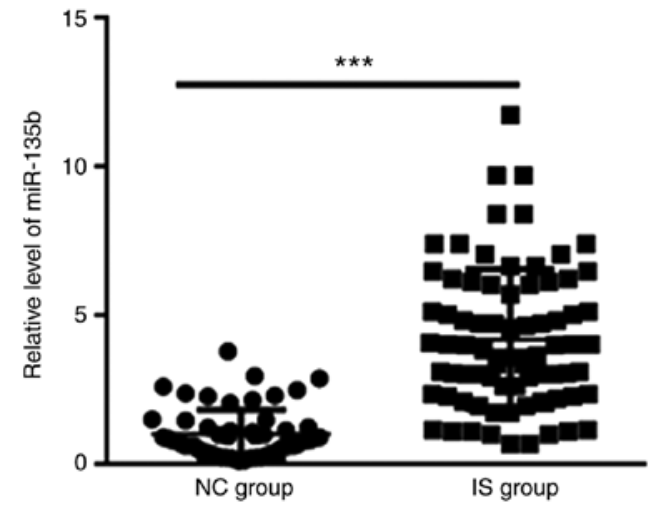

Figure 1. Comparison of miR-135b expression between IS and healthy control group. Reverse transcription-quantitative PCR was performed to analyze the levels of miR-135b in patients with IS and healthy controls. ${ }^{* * *} \mathrm{P}<0.001$. miR-135b, microRNA-135b; IS, ischemic stroke; NC, control.

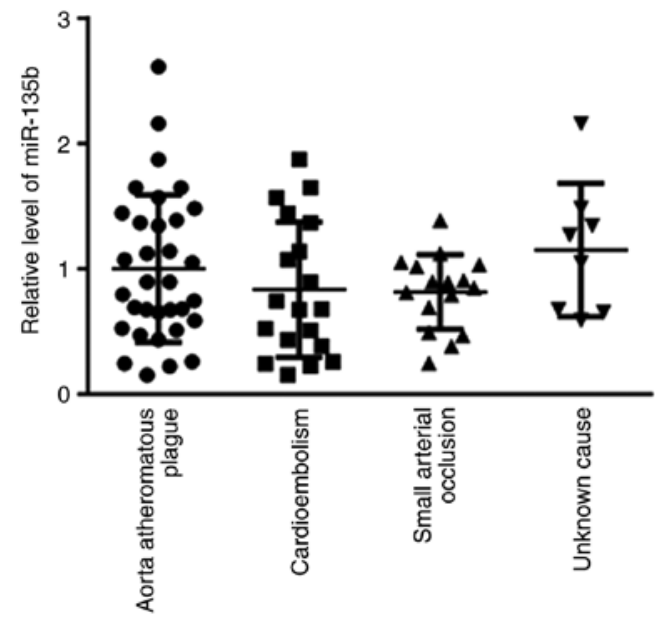

Figure 2. Comparison of miR-135b expression among the four etiological groups of IS with the IS group. No significant difference was observed in expression levels of miR-135b among the aorta atheromatous plague, cardioembolism, small artery occlusion and unknown causes groups. IS, ischemic stroke; miR-135b, microRNA-135b.

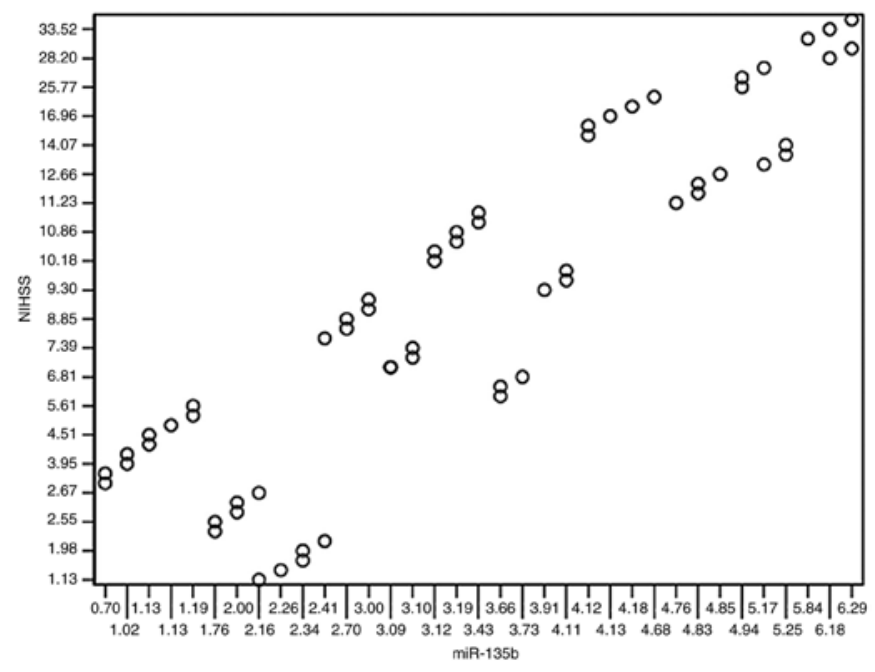

Figure 3. Correlation analysis between peripheral blood miR135b expression and NIHSS scores. Pearson correlation analysis showed that miR-135b correlated positively with NIHSS scores in the peripheral blood of patients in the IS group. IS, ischemic stroke; miR-135b, microRNA-135b; NIHSS, National Institutes of Health Stroke Scale.
Table II. Multivariate logistic regression analysis results.

\begin{tabular}{lccc}
\hline Indicators & HR & $95 \% \mathrm{CI}$ & P-value \\
\hline Hypertension & 2.756 & $1.902-4.321$ & $<0.001$ \\
Hyperglycemia & 1.862 & $1.543-2.356$ & 0.014 \\
Platelet count & 2.094 & $1.678-2.635$ & 0.001 \\
INR & 1.589 & $1.163-2.253$ & 0.025 \\
miR-135b expression & 2.356 & $1.825-3.287$ & 0.002 \\
\hline
\end{tabular}

INR, international normalized ratio; miR-135b, microRNA-135b; $\mathrm{HR}$, hazard ratio, CI, confidence interval.

significant differences were found in the expression levels of miR-135b among the 33 cases of aortic atheromatous plague, 19 cases of cardioembolism, 16 cases of small arterial occlusion and 8 cases with unknown causes (Fig. 2).

Relationship between peripheral blood miR-135b expression levels and NIHSS score. A significant positive correlation was found between miR-135b expression and the NIHSS scores in the peripheral blood of patients in the IS group ( $\mathrm{r}=-0.835$; $\mathrm{P}<0.001$; Fig. 3).

Multivariate logistic regression analysis. Logistic multivariate regression analysis was performed using AIS as the dependent variable, whilst hypertension, hyperglycemia, platelet count, INR and miR-135b expression were used as independent variables. Hypertension, hyperglycemia, platelet count, INR and miR-135b expression were found to be risk factors for AIS, as shown in Table II.

Sensitivity and specificity of peripheral blood miR-135b levels for the diagnosis of IS. The sensitivity and specificity of peripheral blood miR-135b in the diagnosis of IS were analyzed using ROC curve analysis. The AUC of miR-135b was 0.78 (95\% CI, 0.69-0.87; Fig. 4). When the cut-off value was set to $40 \mathrm{fmol} / 1$, sensitivity and specificity of miR-135b for the diagnosis of IS were calculated to be $79.25 \%$ and $64.71 \%$, respectively.

Lower miR-135b expression predicts lower overall survival in patients with AIS. Association between serum miR-135b levels and overall survival rates of patients with AIS was next evaluated. The median value of 2.14 was applied for miR-135b expression in all 76 patients with AIS for separating those with high miR-135b expression $(n=27)$ from low miR-135b expression $(n=49)$. Kaplan-Meier curves indicated that patients with high miR-135b expression exhibited significantly poorer survival compared with those with low miR-135b expression ( $\mathrm{P}=0.032$; Fig. 5).

TRPC6 is a target gene of miR-135b. Based on the aforementioned observations, the potential target genes of miR-135b was subsequently analyzed. Analysis using the TargetScan software identified a conserved binding site in the 3'-UTR of TRPC6 (Fig. 6A). Dual luciferase reporter assay showed that co-transfection with the miR-135b mimic 


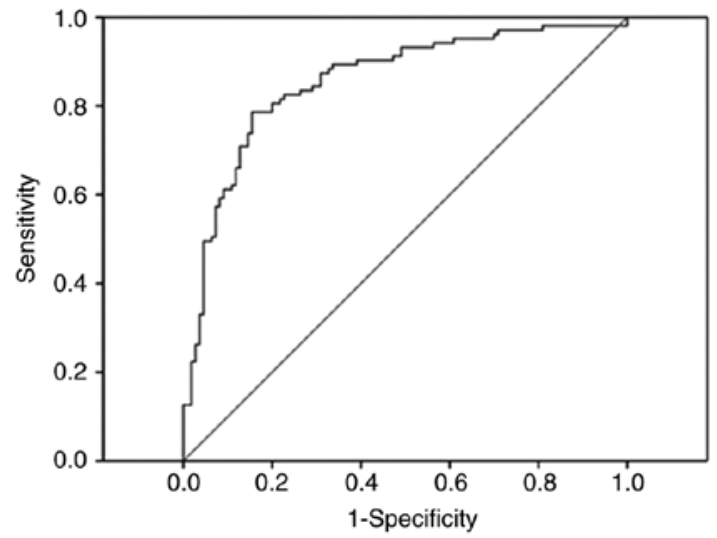

Figure 4. Analysis of diagnostic ability of using miR-135b expression in the peripheral blood for IS. Receiver operating characteristic analysis showed that peripheral blood microRNA-135b could differentiate patients with IS from healthy controls. IS, ischemic stroke.

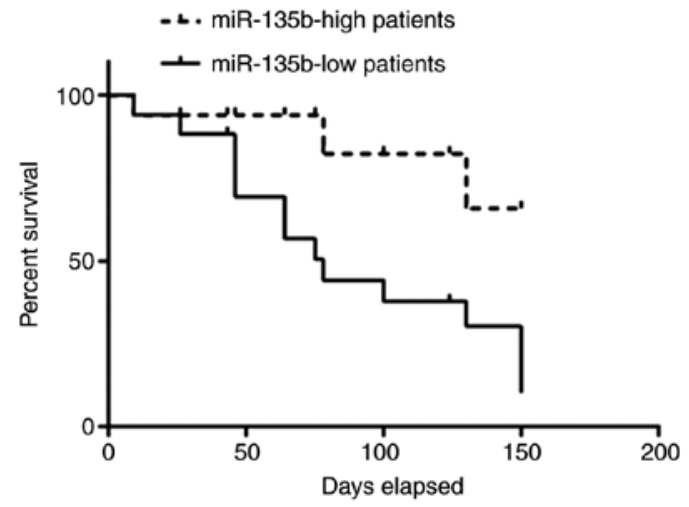

Figure 5. Comparison of overall survival rates between patients with high miR-135b expression and low miR-135b expression. Kaplan-Meier curve analysis suggested that patients with low levels of miR-135b exhibited significantly poorer survival compared with patients with high levels of miR-135b. miR-135b, microRNA-135b.
A

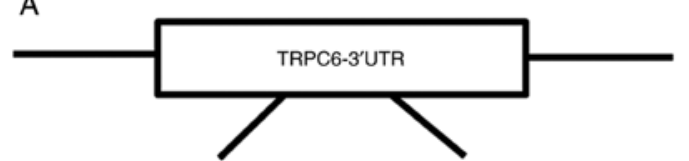

Position 43-50 of TRPC 3'UTR Has-miR-135b

5' UUAUUUGUCCACUUGAAGCCAUA 3' AGUGUAUCCUUACUUUU'C'G'G'U'A'
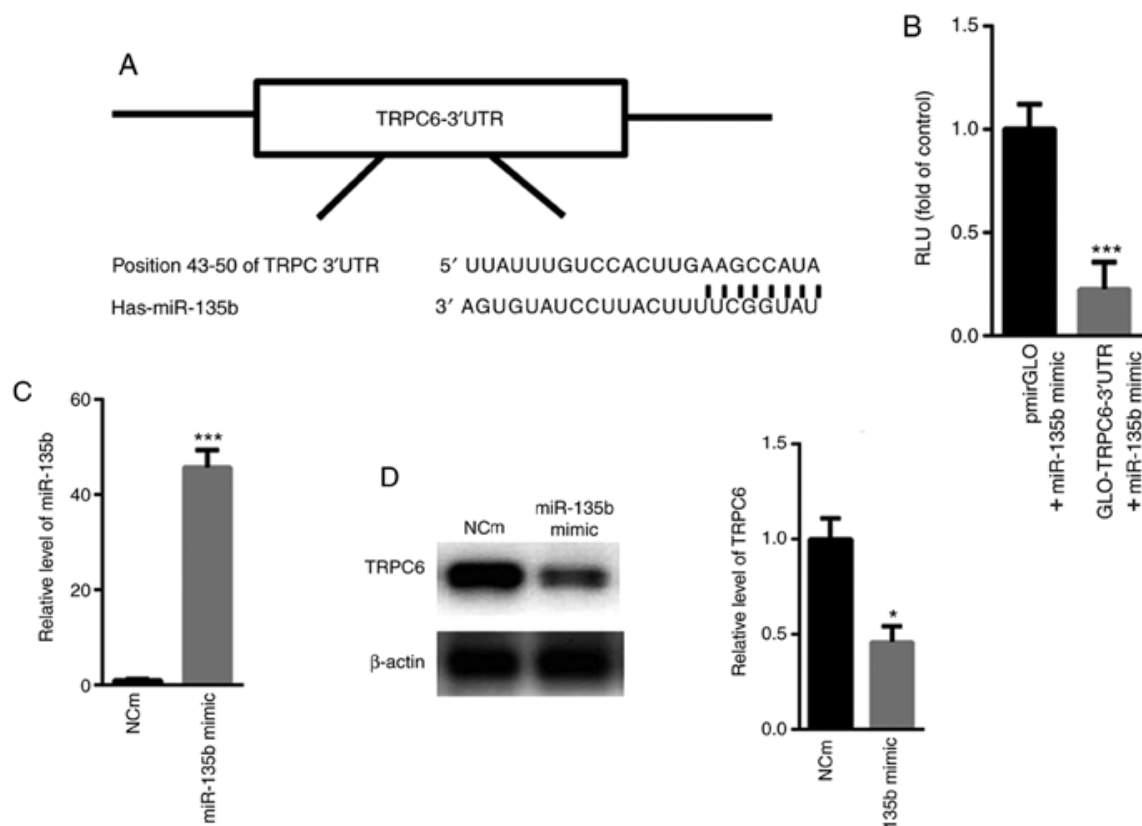

D
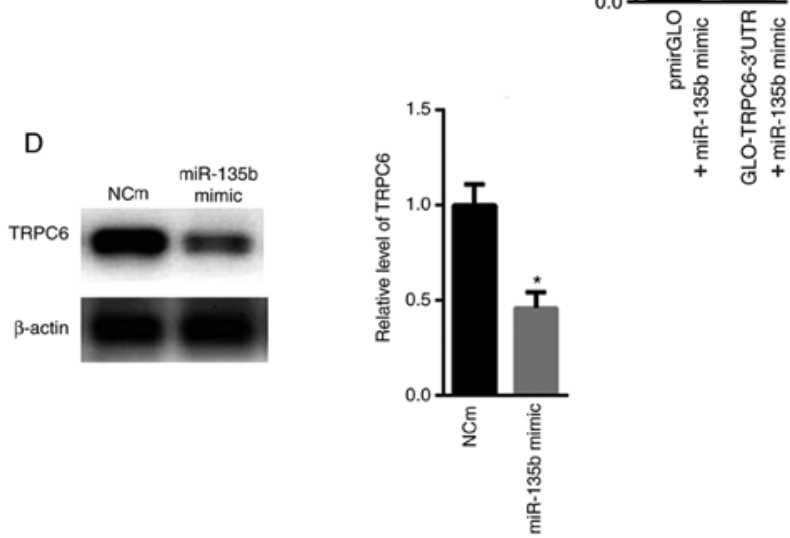

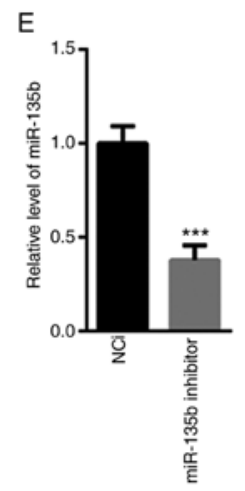

$\mathrm{F}$
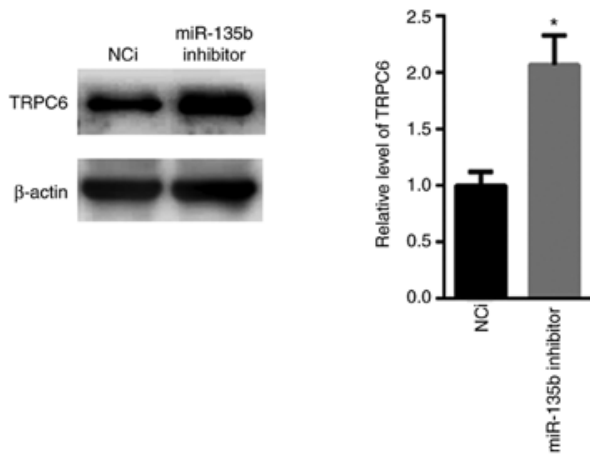

Figure 6. TRPC6 is a target gene of miR-135b. (A) TargetScan analysis identified a conserved binding site for miR-135b in the 3'-UTR of TRPC6. (B) Dual-luciferase assay showed that co-transfection with miR-135b significantly reduced the relative luciferase activity of pmirGLO-TRPC6-3'UTR but not pmirGLO. (C) Transfection with the miR-135b mimic significantly increased the levels of miR-135b and (D) significantly reduced the expression of TRPC6 in PC12 cells compare with the NCm group. (E) Transfection with miR-135 inhibitor significantly reduced the relative expression of miR-135b and (F) significantly increased the expression of TRPC6. ${ }^{*} \mathrm{P}<0.05 ;{ }^{* * *} \mathrm{P}<0.001 .3^{\prime} \mathrm{UTR} ; 3^{\prime}$-untranslated region; NC, negative control transfected with negative control mimics/inhibitor; miR-135b, microRNA-135b; TRPC6, transient receptor potential cation channel subfamily C member 6; RLU, relative luminometer unit; $\mathrm{NCm}, \mathrm{NC}$ mimic; NCi, NC inhibitor. 
significantly reduced the relative luciferase activity of the pmirGLO-TRPC6-3'UTR construct compared with cells co-transfected with the pmirGLO construct (Fig. 6B). Further analysis showed that transfection with the miR-135b mimic significantly increased the levels of miR-135b expression whilst significantly reducing the expression of TRPC6 in PC12 cells, compared with corresponding $\mathrm{NC}$ mimic $(\mathrm{NCm}$; Fig. 6C and D). By contrast, transfection with the miR-135 inhibitor reduced the relative levels of miR-135b expressions whilst enhancing the expression of TRPC6 compared with $\mathrm{NC}$ inhibitor (NCi; Fig. 6E and F). Taken together, these observations indicate that TRPC6 is a target gene of miR-135b.

\section{Discussion}

At present, clinicians place heavy reliance on the presentation of clinical symptoms and signs from patients combined with head imaging to diagnose AIS (23). However, the early symptoms of AIS onset lack specificity and imaging examination is expensive (24), resulting in significant economic burden on patients (25). Therefore, there is a pressing need to explore novel circulating markers for the diagnosis of AIS. Based on TOAST classification, patients with AIS are classified into four categories: Aorta atheromatous plague, cardioembolism, small arterial occlusion and stroke of undetermined etiology (26). However, no significant difference in serum miR-135b levels was identified among the four AIS groups in the present study, indicating that miR-135b is a promising biomarker for AIS but not for a specific subtype of AIS (23). A growing number of studies suggested that changes in miRNA expression in the peripheral blood may be closely associated with the development of AIS, which may be exploited for the development of predictive, diagnostic and prognostic markers for this disease $(24,25)$.

miRNAs are single-stranded RNA molecules 18-25 nucleotides in length that regulate gene expression in many cellular processes $(2,27)$. Although the protective role of miR-135b was previously reported in neurons $(16,17)$, the expression pattern and specific role of miR-135b in AIS remain poorly understood. The present study demonstrated that miR-135b expression in the peripheral blood samples obtained from the IS group was higher compared with the healthy control group. At present, NIHSS is the most commonly used scoring system for stroke severity worldwide (28). The present study found that miR-135b expression levels correlated positively with the NIHSS score in a statistically significant manner, suggesting that miR-135b may be a risk factor the progression of AIS.

The diagnostic value of miR-135 in distinguishing between patients with AIS and healthy controls was subsequently evaluated. The present data showed that the sensitivity and specificity of miR-135b for IS diagnosis revealed an AUC of 0.78 (95\% CI, 0.69-0.87). When the cut-off value was set to $40 \mathrm{fmol} / \mathrm{l}$, the diagnostic sensitivity was $79.25 \%$ and the specificity was $64.71 \%$. These findings suggested that serum miR-135b exhibited a certain accuracy and feasibility as a biomarker of AIS. However, a gap remains between the high specificity and high sensitivity of this marker. Therefore, the combined detection of miRNAs with other protein markers associated with ischemic stroke should be examined in future research.
A number of reports have previously demonstrated that circulating serum miRNAs may serve as potential non-invasive biomarkers for patients with AIS $(5,29)$. For instance, Wang et al (29) reported that serum miR-497 expression was significantly enhanced in patients with AIS compared with healthy controls. Cheng et al (5) suggested that miR-148b-3p, miR-151b and miR-27b-3p may serve as potential biomarkers for diagnosing AIS, with the specific combination of miR-148b-3p and miR-27b-3p being more effective. In accordance with these previous observations, the levels of serum miR-135 in patients with AIS were analyzed based on etiology. Serum miR-135b expression demonstrated positive correlation with the NIHSS scores. However, correlation between serum miR-135b with inflammatory factors, which has been reported to reflect the degree of brain ischemic damage and stroke $(30,31)$, was not investigated in the present study.

The possible target genes of miR-135b were next investigated. TRPC6, an important regulator of the neuronal survival pathway (32), was identified as a possible target gene of miR-135b by bioinformatics analysis. Previous studies have demonstrated the beneficial effects of TRPC6 in protecting against ischemic brain injury (33-35). Guo et al (36) reported that the enhanced expression of TRPC6 reduced neuronal cell death in oxygen glucose-deprived cortical neurons, which serves an important role in combined bone marrow stromal cells (BMSCs) and oxiracetam treatments for cerebral ischemic infarction. A recent report showed that the overexpression of TRPC6 via a CRISPR-based synergistic activation mediator in BMSCs significantly reduced brain injury in rats (33), whilst Li et al (34) demonstrated that TRPC6 overexpression suppressed neurotoxicity and protected neurons from ischemic brain damage. The present study hypothesize that miR-135b may be involved in the process of AIS by targeting the TRPC6 neuronal survival pathway. Therefore, targeting miR-135b may be of potential benefit for stroke prevention and therapy.

It should be noted a number of limitations remain associated with the present study. A time profile of miR-135b would be more practical, which will assist in elucidating the role of miR$135 \mathrm{~b}$ during disease onset and AIS prognosis. However, due to the study design, the number of blood samples obtained remain insufficient. In future studies, a larger cohort of samples should be collected, where miR-135b expression will be studied over a period of time. In addition, the present study mainly focused on measuring the expression of miR-135b in the serum of patients with AIS and to analyze the relationship between its expression levels and the etiology and severity of the disease, thereby investigating miR-135b occurrence in AIS. Investigations into the clinical values of miR-135b in differentiating AIS, hemorrhagic stroke and other brain diseases remain an attractive line of future research. Specifically, samples obtained from patients with other brain diseases could be investigated to elucidate the diagnostic value of serum miR-135b levels further.

In conclusion, miR-135b was found to be closely associated with the severity of patients with AIS in the present study, where high levels of miR-135b in the peripheral blood can be used to indicate the severity of neurological damage and are risk factors for AIS. These findings may contribute to the future clinical diagnosis and prognosis of AIS, however, the importance of miR-135b intervention in the treatment of AIS warrants further investigation. 


\section{Acknowledgements}

Not applicable.

\section{Funding}

The present study was supported by a grant from the Southwest Hospital, Third Military Medical University (grant no. TMMUSH-20150935).

\section{Availability of data and materials}

The datasets used and/or analyzed during the present study are available from the corresponding author on reasonable request.

\section{Authors' contributions}

SY performed the experiments, analyzed the data, wrote the manuscript, designed the experiments, analyzed the data and gave final approval of the version to be published. XYZ, MH, JJW and XMQ performed part of the RT-qPCR experiments. All authors read and approved the final version of the manuscript.

\section{Ethics approval and consent to participate}

The present study was approved by the Research Ethics Committee of Southwest Hospital, Third Military Medical University (Chongqing, China). All patients provided written informed consent.

\section{Patient consent for publication}

Not applicable.

\section{Competing interests}

The authors declare that they have no competing interests.

\section{References}

1. Du K, Zhao C, Wang L, Wang Y, Zhang KZ, Shen XY, Sun HX, Gao W and Lu X: MiR-191 inhibit angiogenesis after acute ischemic stroke targeting VEZF1. Aging (Albany NY) 11: 2762-2786, 2019

2. Chen Z, Wang K, Huang J, Zheng G, Lv Y, Luo N, Liang M and Huang L: Upregulated serum MiR-146b serves as a biomarker for acute ischemic stroke. Cell Physiol Biochem 45: 397-405, 2018.

3. Marquez-Romero JM, Góngora-Rivera F, Hernández-Curiel BC, Aburto-Murrieta Y, García-Cazares R, Delgado-Garzón P, Murillo-Bonilla LM and Ochoa-Solórzano MA: Endovascular treatment of ischemic stroke in a developing country. Vasc Endovascular Surg: Feb 19, 2020 (Epub ahead of print).

4. Béjot Y, Daubail B and Giroud M: Epidemiology of stroke and transient ischemic attacks: Current knowledge and perspectives. Rev Neurol (Paris) 172: 59-68, 2016.

5. Cheng X, Kan P, Ma Z, Wang Y, Song W, Huang C and Zhang B Exploring the potential value of miR-148b-3p, miR-151b and miR-27b-3p as biomarkers in acute ischemic stroke. Biosci Rep 38: 38, 2018.

6. Ji Q, Ji Y, Peng J, Zhou X, Chen X, Zhao H, Xu T, Chen L and $\mathrm{Xu}$ Y: Increased brain-specific MiR-9 and MiR-124 in the serum exosomes of acute ischemic stroke patients. PLoS One 11: e0163645, 2016.

7. Jia L, Hao F, Wang W and Qu Y: Circulating miR-145 is associated with plasma high-sensitivity C-reactive protein in acute ischemic stroke patients. Cell Biochem Funct 33: 314-319, 2015.
8. Jiang M, Wang H, Jin M, Yang X, Ji H, Jiang Y, Zhang H, Wu F, Wu G, Lai X, et al: Exosomes from MiR-30d-5p-ADSCs reverse acute ischemic stroke-induced, autophagy-mediated brain injury by promoting M2 microglial/macrophage polarization. Cell Physiol Biochem 47: 864-878, 2018.

9. Jin F and Xing J: Circulating miR-126 and miR-130a levels correlate with lower disease risk, disease severity, and reduced inflammatory cytokine levels in acute ischemic stroke patients. Neurol Sci 39: 1757-1765, 2018.

10. Khanna S, Rink C, Ghoorkhanian R, Gnyawali S, Heigel M, Wijesinghe DS, Chalfant CE, Chan YC, Banerjee J, Huang Y, et al: Loss of miR-29b following acute ischemic stroke contributes to neural cell death and infarct size. J Cereb Blood Flow Metab 33: 1197-1206, 2013.

11. Li SH, Chen L, Pang XM, Su SY, Zhou X, Chen CY, Huang LG, Li JP and Liu JL: Decreased miR-146a expression in acute ischemic stroke directly targets the Fbxl10 mRNA and is involved in modulating apoptosis. Neurochem Int 107: 156-167, 2017.

12. Su ZF, Sun ZW, Zhang Y, Wang S, Yu QG and Wu ZB: Regulatory effects of $\mathrm{miR}-146 \mathrm{a} / \mathrm{b}$ on the function of endothelial progenitor cells in acute ischemic stroke in mice. Kaohsiung J Med Sci 33: 369-378, 2017.

13. Tiedt S, Prestel M, Malik R, Schieferdecker N, Duering M, Kautzky V, Stoycheva I, Böck J, Northoff BH, Klein M, et al: RNA-Seq identifies circulating miR-125a-5p, miR-125b-5p, and miR-143-3p as potential biomarkers for acute ischemic stroke. Circ Res 121: 970-980, 2017.

14. Wang Y, Zhang Y, Huang J, Chen X, Gu X, Wang Y, Zeng L and Yang GY: Increase of circulating miR-223 and insulin-like growth factor-1 is associated with the pathogenesis of acute ischemic stroke in patients. BMC Neurol 14: 77, 2014.

15. Wu J, Du K and Lu X: Elevated expressions of serum miR-15a, miR-16, and miR-17-5p are associated with acute ischemic stroke. Int J Clin Exp Med 8: 21071-21079, 2015.

16. Zhang J, Liu W, Wang Y, Zhao S and Chang N: miR-135b plays a neuroprotective role by targeting GSK3 $\beta$ in $\mathrm{MPP}^{+}$-intoxicated SH-SY5Y Cells. Dis Markers 2017: 5806146, 2017.

17. Sillivan SE, Jamieson S, de Nijs L, Jones M, Snidjers C, Klengel T, Joseph NF, Krauskopf J, Kleinjans J and Vinkers CH: MicroRNA regulation of persistent stress-enhanced memory. Mol Psychiatry: May 29, 2019 (Epub ahead of print).

18. Jiang B, Ball RL, Michel P, Jovin T, Desai M, Eskandari A, Naqvi $\mathrm{Z}$ and Wintermark $\mathrm{M}$ : Prevalence of imaging biomarkers to guide the planning of acute stroke reperfusion trials. Stroke 48: 1675-1677, 2017.

19. Kwah LK and Diong J: National Institutes of Health Stroke Scale (NIHSS). J Physiother 60: 61, 2014.

20. Livak KJ and Schmittgen TD: Analysis of relative gene expression data using real-time quantitative PCR and the $2^{-\Delta \Delta C T}$ Method. Methods 25: 402-408, 2001.

21. Yang X, Liu Y, Liu C, Xie W, Huang E, Huang W, Wang J, Chen L, Wang H, Qiu P, et al: Inhibition of ROCK2 expression protects against methamphetamine-induced neurotoxicity in PC12 cells. Brain Res 1533: 16-25, 2013.

22. Rong F, Gao X, Liu K and Wu J: Methotrexate remediates spinal cord injury in vivo and in vitro via suppression of endoplasmic reticulum stress-induced apoptosis. Exp Ther Med 15: 4191-4198, 2018.

23. Xiang W, Tian C, Lin J, Wu X, Pang G, Zhou L, Pan S and Deng Z: Plasma let-7i and miR-15a expression are associated with the effect of recombinant tissue plasminogen activator treatment in acute ischemic stroke patients. Thromb Res 158: $121-125,2017$.

24. Zhao B, Zhu Z, Hao J, Wan Z and Guo X: Decreased plasma miR-335 expression in patients with acute ischemic stroke and its association with calmodulin expression. J Int Med Res 44: 1331-1338, 2016.

25. Zhou J, Chen L, Chen B, Huang S, Zeng C, Wu H, Chen C and Long F: Increased serum exosomal miR-134 expression in the acute ischemic stroke patients. BMC Neurol 18: 198, 2018.

26. Ko Y, Lee S, Chung JW, Han MK, Park JM, Kang K, Park TH, Park SS, Cho YJ, Hong KS, et al: MRI-based algorithm for acute ischemic stroke subtype classification. J Stroke 16: 161-172, 2014.

27. Shi FP, Wang XH, Zhang HX, Shang MM, Liu XX, Sun HM and Song YP: MiR-103 regulates the angiogenesis of ischemic stroke rats by targeting vascular endothelial growth factor (VEGF). Iran J Basic Med Sci 21: 318-324, 2018.

28. Yao S, Tang B, Li G, Fan R and Cao F: miR-455 inhibits neuronal cell death by targeting TRAF3 in cerebral ischemic stroke. Neuropsychiatr Dis Treat 12: 3083-3092, 2016. 
29. Wang J, Lin M, Ren H, Yu Z, Guo T and Gu B: Expression and clinical significance of serum miR-497 in patients with acute cerebral infarction. Clin Lab 65: 65, 2019.

30. Cojocaru IM, Cojocaru M, Tănăsescu R, Iliescu I, Dumitrescu L and Silosi I: Expression of IL-6 activity in patients with acute ischemic stroke. Rom J Intern Med 47: 393-396, 2009.

31. Kwan J, Horsfield G, Bryant T, Gawne-Cain M, Durward G, Byrne CD and Englyst NA: IL-6 is a predictive biomarker for stroke associated infection and future mortality in the elderly after an ischemic stroke. Exp Gerontol 48: 960-965, 2013.

32. Griesi-Oliveira K, Acab A, Gupta AR, Sunaga DY, Chailangkarn T, Nicol X, Nunez Y, Walker MF, Murdoch JD, Sanders SJ, et al: Modeling non-syndromic autism and the impact of TRPC6 disruption in human neurons. Mol Psychiatry 20: 1350-1365, 2015

33. Li W, Yang F, Gao J, Tang Y, Wang J and Pan Y: Over-expression of TRPC6 via CRISPR based synergistic activation mediator in BMSCs ameliorates brain injury in a rat model of cerebral ischemia/reperfusion. Neuroscience 415: 147-160, 2019.
34. Li H, Huang J, Du W, Jia C, Yao H and Wang Y: TRPC6 inhibited NMDA receptor activities and protected neurons from ischemic excitotoxicity. J Neurochem 123: 1010-1018, 2012.

35. Du W, Huang J, Yao H, Zhou K, Duan B and Wang Y: Inhibition of TRPC6 degradation suppresses ischemic brain damage in rats. J Clin Invest 120: 3480-3492, 2010.

36. Guo C, Ma Y, Ma S, Mu F, Deng J, Duan J, Xiong L, Yin Y, Wang Y, Xi M, et al: The role of TRPC6 in the neuroprotection of calycosin against cerebral ischemic injury. Sci Rep 7: 3039, 2017.

This work is licensed under a Creative Commons Attribution-NonCommercial-NoDerivatives 4.0 International (CC BY-NC-ND 4.0) License. 